\title{
Attention Control during Distance Learning Sessions
}

\author{
Giuseppe Mastronardi, Vitoantonio Bevilacqua, \\ Roberto Fortunato Depasquale, and Massimiliano Dellisanti Fabiano Vilardi \\ Politecnico di Bari, Bari, Italy \\ \{mastrona, bevilacqua, m.dellisantifabiano\}@poliba.it, \\ \{robertof ortunato.depasquale\}@gmail.com
}

\begin{abstract}
The distance learning (DL) is a teaching system that extends the education beyond the physical barriers, providing access to remote places and disabilities. The increasing need of procedures for DL certification is now involving biometric approach. An analysis of biometric techniques is shown in order to ensure the users authentication, to verify the individual's attention level and then to certificate the learning outcomes. That is necessary to implement a system to identify uniquely the users and to track both path's carried (visited pages) and use's time, to have a secure users identification and also validation of the environments conditions in which they take place during possible tests of certification. The appropriate biometric technique is appeared the Face Recognition because it allows a real-time verification of the real presence, low implementation costs by use of webcam and reasonable degree of reliability. To avoid the influence related to environmental conditions, it has been realized a modular system that implements Detection and Recognition operations. The implemented system is able to verify the presence of learners beyond the screen during lessons or learning tests, to allow authentication and to verify the simultaneous presence of other individuals in order to start an alarm if unregistered peoples are present during learning or testing sessions. This system is also capable to recognize the attention level of users through Request Random Windows (RRW). The application opens casually a RRW in different screen position during the DL and asks learner to click upon to close it within a few seconds. When this window is closed, a new step of Face Recognitions is performed again to validate the presence of the same user. Interesting results are obtained in experimental cases employing these techniques on a individuals samples set.
\end{abstract}

Keywords: attention control, distance learning, biometric techniques.

\section{Introduction}

New communication technologies (synchronous and asynchronous) are pushing to processes' development of learning and training. Today, it's possible to access training courses online through Internet connections and webcams. These

A. Petrosino, L. Maddalena, P. Pala (Eds.): ICIAP 2013 Workshops, LNCS 8158, pp. 545-549, 2013.

(C) Springer-Verlag Berlin Heidelberg 2013 
technology supports are exploited interactive learning processes, facilitated by videos, quizzes and teleconferencing. In fact, the webcam can be used to recognize the DL's target users through biometric techniques. The scene analysis is crucial: a context, where more subjects are captured via a webcam (with low definition), highlights conditions less facilitating for face-detection. Using optimal environmental conditions of scenes (no more individuals, uniform and not white background) allows more precise face-detection [1] 3] and face-recognition [2] 3] (with a positive identification if a person's face belongs at a white-list). Then, to verify the continuous presence of the subject during communication and their attention level degree, there are required processes of face-recognition in both periodic and randomized way, depending to the confirm request upon windows appearing in random moments and in random screen positions.

\section{Paper Preparation}

In order to make the system more manageable and modular, the MVC pattern (Model-View-Controller) has been adopted. In this approach, the design of a web application is divided into three levels: the Presentation Layer (View) that implements modes and forms of data presentation to the user; the Application Logic Layer (Model), that interfaced the database with the procedures to extract and to process data (templates of faces and reaction times); the Control Layer (Controller) that implements the interactions between the other layers.

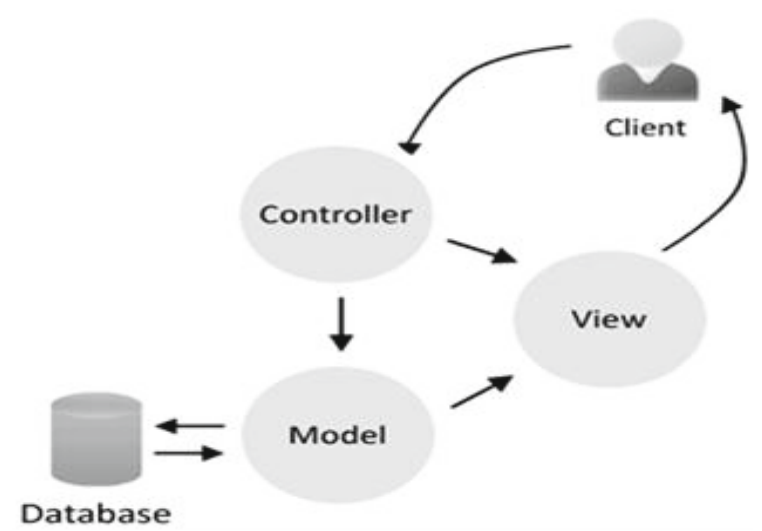

Fig. 1. MVC pattern (Model-View-Controller)

The recognition process must be based on an enrollment phase. This phase is characterized by: acquisition subject face; extraction subject features; saving obtained template; combining templates to respective individual data.

The proposed system is characterized by a step of pre-processing in which the image is processed by two subsystems connected in cascade: Haarcascade 


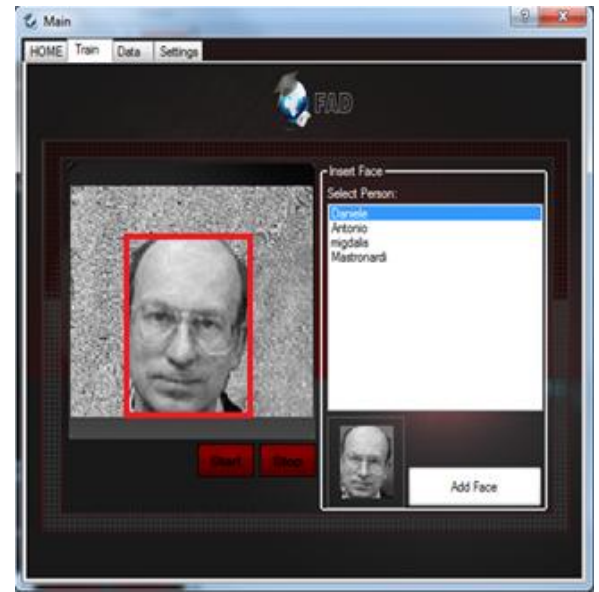

Fig. 2. Enrollment phase with face-detection and face characterization processes

2] 5] and than Principal Component Analysis (PCA) 6]. After the real-time acquisition of an image by webcam, the algorithm Haarcascade processes the one to detect the presence of the human face returning an image containing only one face. From the subsequent PCA application, a face image is obtained as face projection within the sub-space (face-space). This projection is saved in a database and then associated with the individual data. From the training-set (a training set consisting of all the known samples), containing the location in the subspace of all subjects, it will be possible to recognize the individual with the Eigenfaces method. The login phase involves the insertion of the username, the password and the verification of the associated individual face. After the recognition, the subject can access at the DL and attend the lesson. During the DL session, in random mode, checks are performed on the verification of the attention level. The application opens casually a Request Random Windows (RRW) in different screen position during the DL and asks learner to click upon to close it within a few seconds. The method used to calculate the attention degree is to record the reaction time. The reaction time is the time between appearance of the RRW and the mouse click on its to confirm the request.

When this window is closed, a new step of Face Recognitions is performed again to validate the presence of the same user. The training session (or test) is suspended if no face is present or it is not the same as starting. At the same way, the training session is suspended if the permissible response time exceeds the time threshold (time-out). Obviously, the user can pause the DL at any time.

\section{Processing}

The testing phase of the system has allowed to identify the ideal conditions of the PCA method, working on the number of components in order to improve the biometric classification. The considered images represent a small sample of 


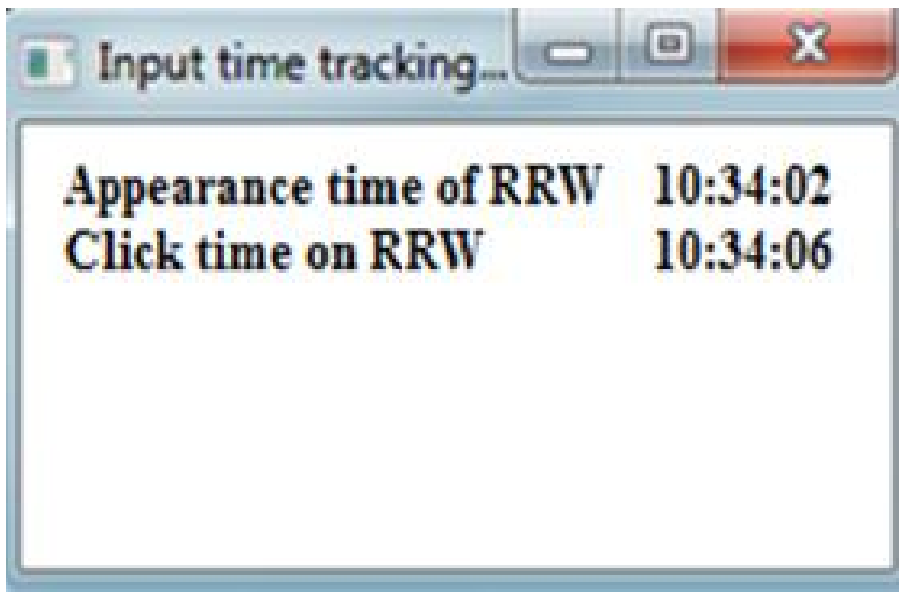

Fig. 3. Data on the reaction time of an experimental case

actual individuals. Each of them is asked for repeated exposure to the system for a period of sixty seconds in order to evaluate the recognition rate vs time of recognition $\operatorname{tr}$ (time of real recognition) and the exposure time te (time period of acquisition):

$$
P_{r}=t_{r} / t_{e}
$$

By way of example, the figure to right shows the rate of recognition in four individual experiment by varying the number of images (Eigenfaces). It's possible to show how the optimal value corresponds to 15 snapshots to get the best performance. Therefore, a greater number of Eigenfaces may be considered redundant.

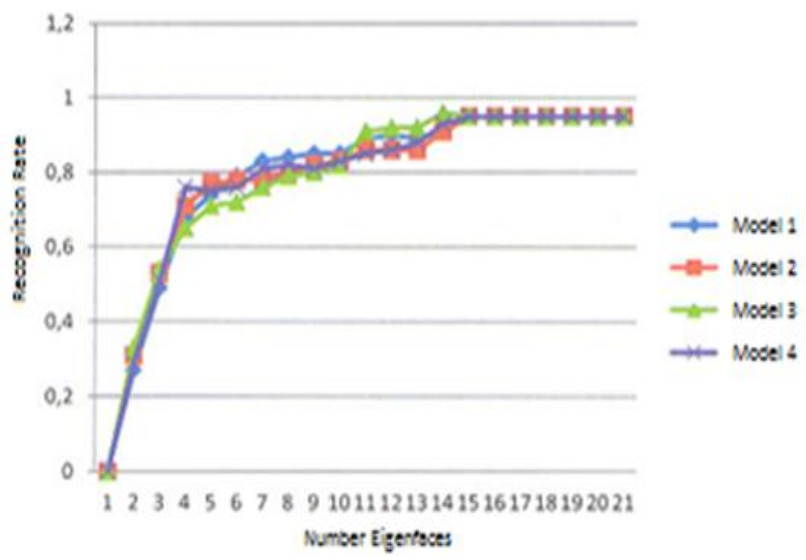

Fig. 4. Recognition Rate of 4 models included in a experimental case 


\section{Conclusions}

Distance learning systems are in continuous evolutions and diffusion. In fact, the classic educational process is giving way to remote innovative systems to meet the expectations of users spread over a large geographical area, but also to satisfy the same education providers, who need to reduce the cost of location management and maintenance. The training online is growing everywhere and is therefore a reality that sees a growing involvement of biometrics for student identification. The biometric facial recognition, combined with response times to the requests for attention by RRW, allowed us to develop a valuable tool for secure authentication of individuals during the administration of a DL module. Therefore, this hybrid approach becomes strongly recommended in all those systems that require a minimum level of security to avoid misleading identity. The use of biometrics of face, less intrusive and more efficient, can be used in any structure with easy possibility to control lighting conditions and acquisition distance. The presented application is a prototype platform in continuous development. In this paper, an analysis was made of the state of art and technology related to the use of certain techniques biometrics, illustrating a possible application scenario that may also involve the subsequent possible process of testing sessions.

\section{References}

1. Sakai, T., Nagao, M., Fujibayashi, S.: Line Extraction and Pattern Detection in a photograph. Pattern Recognition 1(3), 233-248 (1969)

2. Kanade, D.: Computer Recognition of Human Faces. Birkauser, Basel and Stuttgart (1997)

3. Reifeld, T., Yeshurun, Y.: Robust Detection of Facial Features by Generalized Symmetry. In: Proceedings of 11th International Conference on Pattern Recognition (1992)

4. Bradski, G., Kaehler, A.: Learning: OpenCV. Computer Vision with the OpenCV Library. O'Reilly Media (2008)

5. Padilla, R., Costa Filho, C.F.F., Costa, M.G.F.: Evaluation of Haar Cascade Classifiers Designed for Face Detection. In: World Academy of Science, Engineering and Technology (2012)

6. Turk, M., Pentland, A.: Eigenfaces for Recognition. Journal of Cognitive Neuroscience 3(1), 71-86 (1991) 